\section{Technologie reguliert Recht?}

M.R. McGuire: Technology, Crime and Jus-
tice: The Question Concerning Technomia.
London et al.: Routledge 2012, 284 S.,
ISBN 978-1-84392-857-7, Euro 112,46

\section{Rezension von Christina Merz, ITAS}

Michael McGuire, derzeit tätig als Senior Lecturer am Soziologischen Institut der Universität Surrey, Großbritannien, untersucht in seinem 2012 erschienenen Buch die Rolle von Technik und Technologie in ihrer Bedeutung für Kriminalität, Kriminalitätskontrolle und Strafverfolgung. Dabei zielt er bewusst darauf ab, Kernfragen zu dieser Thematik losgelöst von speziellen Forschungsrichtungen und Techniken, wie zum Beispiel jenen zu Internetkriminalität und Überwachung, auf wissenschaftlich-theoretische Weise zu erörtern. Beginnend mit der Frage nach der Definition von Technik und Technologie und wie sich diese in ihrer Bedeutung für den kriminellen und strafrechtlichen Kontext historisch entwickelt haben, wendet sich der Autor im Hauptteil des Buches einzelnen Technologien und Techniken (z. B. Informations- und Kommunikationstechnologien, Biotechnologien) zu. Dabei ist das Buch zumeist in drei Betrachtungsebenen gegliedert: Die Frage nach der Regulierung aufgrund des Missbrauchs von Technologie (Straftaten), die Frage nach der Regulierung mittels Technologie (Kriminalitätsprävention) und jene Frage nach der Regulierung des Rechts und der Rechtssysteme durch Technologie.

\section{Reguliert von, mit und aufgrund von Technologie}

Wendet man sich dem Buch mit der Absicht zu, Antworten zur Rolle von Technologien im kriminellen Kontext und zum Ausmaß der Ausnutzung von Technologie für Straftaten zu finden, so wird man schnell feststellen, dass der Autor dieser Erwartung zwar Rechnung trägt, den Bogen jedoch deutlich weiter spannt.

Schon das Kapitel zu Informations- und Kommunikationstechnologien ist zwar hinsichtlich einzelner Straftaten und Straftatbestände gegliedert, der Fokus jedoch - und das ist das eigentlich Interessante dabei - liegt auf der ungeklärten Frage, warum gerade diese Technologien in den Mittelpunkt des Interesses mehrerer gesellschaftlicher Teilbereiche, wie zum Beispiel der Justiz, Politik und Wissenschaft gerückt sind.

Die Aussage ,[...] there seems nothing in what this technology does that is ultimately unique" (S. 80) ist dabei sicherlich provokant. Sie verleitet jedoch tatsächlich zur Frage, weshalb gerade Kriminalität in Verbindung mit diesen Technologien eine eigene Etikettierung als „cybercrime“ oder „Internetkriminalität" erhalten hat und welchen Erkenntnisgewinn man daraus ziehen kann. Nach Meinung des Autors trägt dies nicht zur Antwort auf die Frage, ,wie“ Informations- und Kommunikationstechnologien Kriminalität fördern bei. Mehr noch: Laut McGuire führt dies weg von den wichtigen Fragen nach sozialen Veränderungen, die mit dieser Art von Technologie einhergehen. Die Verwischung der Grenzen zwischen Raum und Zeit im sozialen Gefüge oder die Veränderung unseres Verständnisses von Wertigkeit - weg von fassbaren Artefakten (z. B. Geldbörse) hin zum Zugang zu Informationen und Daten (z. B. Authentifizierungscodes) - werden dabei als Beispiele genannt. Zusätzlich zur Ausblendung der sozialen Veränderungen jedoch, führe der Begriff „cybercrime“ zur Wahrnehmung einer Grenze zwischen realer und virtueller Welt und der Annahme, informations- und kommunikationstechnologisch gestützte Kriminalität passiere nur innerhalb des Internets. Letzteres, so der Autor, begünstige das Übersehen aktueller Missbrauchspotenziale (z. B. mittels Smartphones) und verschleiere den Blick auf den Umgang mit der Technologie selbst.

Damit schlägt McGuire die Brücke zum nächsten Kapitel, das sich mit der Regulierung von Informations- und Kommunikationstechnologien befasst. Er geht davon aus, dass Informations- und Kommunikationstechnologien seitens der rechtssprechenden und rechtsdurchsetzenden Institutionen in einem hohen Maße anerkannt sind und zu einer Veränderung der Polizei- und Justizarbeit geführt haben. Dies erinnert stark an eine im englischsprachigen Raum geführte Debatte, die sich mit der Frage beschäftigt, ob 
die erklärende, nach den Ursächlichkeiten kriminellen Verhaltens suchende Kriminologie durch die sog. „crime science“ abgelöst werden sollte. Letztere befasst sich u. a. mit der technologisch gestützten Prävention und Kriminalitätskontrolle (z. B. Video-Überwachung, Hot-Spot-Datamining), um die Möglichkeiten kriminellen Verhaltens im Voraus zu mindern oder Straftaten schneller aufzuklären. Dadurch werde, so die Argumentation, die Effektivität in der Kriminalitätsbekämpfung erhöht (s. Clarke 2004). McGuire hingegen sieht in dieser Entwicklung eher eine Durchdringung des Rechtssystems mit technologischen Normen, eine These, die er auch im anschließenden Kapitel bezüglich Kriminalität und Kriminalitätskontrolle mittels Bio-, Chemie- und Nanotechnologien weiter verfolgt.

McGuire spannt den Bogen weit, indem er - beginnend mit dem Ersten Weltkrieg - verschiedene Möglichkeiten des Missbrauchs biologischer und chemischer Technologien in unterschiedlichen Bereichen (vom militärischen Einsatz hin zur Spielzeug-, Lebensmittel- und Pharmaindustrie) darstellt. Zentraler Punkt ist dabei die Kritik an kollektiven Akteuren (Staaten oder „big (bad) pharma“", S. 131) und der seiner Meinung nach unzureichenden Strafverfolgung in diesem Bereich. Gleichsam deutet er in diesem Kapitel jedoch auch auf die unterschätzten Potenziale neuer Technologien (z. B. im Nanobereich) hin, deren kriminogenes Potenzial weitestgehend unbekannt ist.

Im Kapitel „micro-control“ formuliert er die These der voranschreitenden Regulierung der Gesellschaft seitens technologischer Normen weiter aus. Insbesondere forensische Methoden hätten zu einer Veränderung des Strafverfolgungssystems geführt. Und so wird nicht nur die Fehleranfälligkeit dieser Methoden kritisiert, sondern mehr noch das hohe Vertrauen, das in forensische oder medizinische Experten während eines Gerichtsprozesses gesetzt wird. Technologische Normen - repräsentiert von sog. „expert witnesses“ (S. 141) und basierend auf Wahrscheinlichkeitshypothesen und messbaren Indikatoren - dominierten den Gerichtsprozess der heutigen Zeit. Auch wenn McGuire sich hier auf die strafrechtliche Beweisführung bezieht, so mag manch ein Leser an dieser Stelle des Buches durchaus ein mulmiges Gefühl bekommen und an die frühe positivistische Schule der Kriminologie des 19. Jahrhunderts (zumindest teilweise) erinnert werden. Damals wurde versucht, die Wahrscheinlichkeit kriminellen Verhaltens an biologischen Merkmalen (Gesichtszüge, abstehende Ohren etc.) festzumachen.

Im vorerst letzten Kapitel wendet sich McGuire Alltagsgegenständen (z. B. Messer aber auch Bügelbrett) zu. Er hinterfragt, weshalb Straftaten mittels dieser Gegenstände nicht wie zum Beispiel „Internetkriminalität“ durch das verwendete Werkzeug rechtlich und kriminologisch definiert sind, obwohl diese - ähnlich der vorher besprochenen Technologien - ebenfalls eine Erweiterung der menschlichen Fähigkeiten darstellen. Eine abschließende Antwort hierauf vermag der Autor in diesem Kapitel jedoch nicht zu geben.

Mit der Benennung des Grundes der voranschreitenden Dominanz technologischer Normen im Kapitel „Technology, science and justice" schließt McGuire das Buch weitestgehend $\mathrm{ab}$, indem er den Wissenschaftsglauben und das Vertrauen in wissenschaftliche Innovationen und deren zugeschriebenen Attribute (z. B. Effizienz) als Einflussfaktoren für diese Entwicklung verantwortlich macht. Mit der Unterwanderung des (Straf-)Rechts und der Rechtsausübung seitens der Technologie, und damit auch seitens der Wissenschaft, verliert das Recht als eigene Instanz seiner Meinung nach mehr und mehr an Bedeutung. Abhilfe, so der Autor, könne hier nur eine offene Diskussion um die Regulierung von Technologie aber auch um die Regulierung durch Technologie (z. B. in der Kriminalprävention) schaffen. McGuire schlägt vor, einen partizipativen Mechanismus zu entwickeln, der statt „Top-Down-Governance“ offen für eine pluralistischere, bürgerliche Kontrolle sei.

\section{Von DNA-Analysen zu Schönheits-OPs und südafrikanischen Sprintern}

Bereits mehrfach angedeutet wurde, dass McGuire einen weiten Bogen um die Thematik „Technologie, Kriminalität und Recht“" spannt. Dies wird schon in den ersten Kapiteln deutlich. Hier versucht McGuire zunächst Technik als sol- 
che zu fassen und zu definieren. Durch Rückgriff auf Heidegger, Latour und andere, begreift er Technik als etwas Soziales und fügt hinzu, dass diese unsere Fähigkeiten ausweite und uns befähige, unsere Reichweite über Raum und Zeit hinaus auszudehnen. Gleichsam zeichnet er eine historische Linie von prämodernen Techniken zu Technologien der Neuzeit.

Dieses breite Verständnis führt jedoch im weiteren Verlauf des Buchs zuweilen zu einem Verlust des im Klappentext versprochenen Fokus. So muss sich der Leser sodann plötzlich mit den regulatorischen Problemen im Bereich des Behindertensports und der kosmetischen Chirurgie auseinandersetzen, da sowohl Carbon-Prothesen als auch Brustimplantate unter die Definition des Autors von Technik und Technologie als Ausweitung und Aufwertung menschlicher Fähigkeiten fallen.

Eine ähnliche Verwirrung des Lesers vermag sich auch bei der Verwendung des Begriffes ,justice“ einstellen. Kann dieser Begriff in der Übersetzung des Englischen ins Deutsche zwar unterschiedlich von Recht hin zu Gerechtigkeit konnotiert werden, so wird er in der Inhaltsbeschreibung des Buches klar mit „,criminal justice“, der Strafrechtspflege, gleichgesetzt. Obwohl McGuire zwar schon zu Beginn des Buches darauf aufmerksam macht, dass er zuweilen auch einen weiteren Begriff von ,justice“ verwendet (S. 29), so bleibt es fraglich, ob Rückgriffe auf andere Rechtsbereiche und Risiken (z. B. verbraucherschutzrelevante Regularien oder Gesundheitsrisiken bei der Bedienung von Touchscreens) zur Unterstützung seiner These der Dominanz technologischer Normen wirklich beitragen.

\section{Fazit}

Zumeist präzise erläutert und mit Beispielen unterlegt, wirft der Autor wichtige Kernfragen über die Beziehung von Technologie, Kriminalität und Recht losgelöst von bestimmten Straftaten auf, die bis heute sowohl im wissenschaftlichen als auch gesellschaftlichen Diskurs unzureichend behandelt worden sind. Besonders interessant ist dabei die Frage nach der Verquickung von Recht beziehungsweise Justiz und
Wissenschaft. Ob man McGuires These - Technologien halten aufgrund der positiven Attribute, die der Wissenschaft zugeschrieben werden Einzug in den Gerichtssaal - Folge leisten will, bleibt dahingestellt. Jedoch wird damit eine nicht unwesentliche Frage aufgegriffen, die bereits 2005 in einer Veröffentlichung von Sheila Jasanoff mit dem Titel „Law's Knowledge“ zu eben dieser Beziehung des Rechts und der Wissenschaft gestellt wurde. Basierend auf der Kritik am „Daubert-Standard“ des US-amerikanischen Gerichtswesens, der es - verkürzt gesprochen - der Bewertung des Richters überlässt, ob ein Beweis wissenschaftlichen Grundprinzipien genügt, um im Prozess zugelassen zu werden, kritisiert Jasanoff ähnlich McGuire die simple Übertragung wissenschaftlicher Standards auf gerichtliche Entscheidungsprozesse.

Lässt man das Abgleiten des Fokus, das sich aufgrund der Breite der Definitionen und Beispiele zeitweise einstellt, beiseite, so bietet McGuires Buch einen interessanten Überblick über die Problemstellungen im Verhältnis von Technologie, Kriminalität und Recht. Zwar werden durch die wissenschaftlich-theoretische Herangehensweise mehr Fragen aufgeworfen als Antworten gegeben, die Relevanz dieser Fragen erscheint jedoch unbestreitbar. Auch bleibt es letztlich dahingestellt, ob man des Autors These, technologische Normen durchdringen mehr und mehr unser Rechtssystem, in seiner Absolutheit Folge leisten möchte. Die Beispiele, die McGuire nennt, regen jedoch durchaus zum Nachdenken an und zeigen auf, dass im Verhältnis zwischen Technologie, Kriminalität und Recht noch einiges an Forschungsarbeit zu leisten ist.

\section{Literatur}

Clarke, R., 2004: Technology, Criminology and Crime Science. In: European Journal on Criminal Policy and Research 10/1 (2004), S. 55-63

Jasanoff, S., 2005: Law's Knowledge: Science for Justice in Legal Settings. In: American Journal of Public Health 95/Suppl. 1 (2005), S. S49-S58 